\title{
Integration and Clinical Evaluation of an Interactive Controllable Robotic System for Anaplastology
}

\author{
Andreas Hein ${ }^{1}$, Martin Klein ${ }^{2}$, Tim C. Lueth ${ }^{1}$, Jochen Queck ${ }^{1}$, Malte Stien ${ }^{1}$, \\ Olaf Schermeier ${ }^{1}$, Juergen Bier ${ }^{2}$ \\ ${ }^{1}$ Berlin Center for Mechatronic Medical Devices \\ Fraunhofer IPK - Charité • Campus Virchow, Clinic for Oral and Maxillofacial Surgery \\ Augustenburger Platz 1, 13353 Berlin \\ \{andreas.hein, tim.lueth, jochen.queck, malte.stien, \\ olaf.schermeier\}@charite.de \\ http://www. charite.de/rv/mkg/srl/ \\ ${ }^{2}$ Clinic for Oral and Maxillofacial Surgery \\ Charité, Campus Virchow, D-13353 Berlin, Germany \\ \{martin.klein, juergen.bier\}@charite.de \\ http://www. charite.de/rv/mkg/
}

\begin{abstract}
In this paper the integration and evaluation of a robotics system developed at the Surgical Robotics Lab at the Charité, Berlin is presented. In the first clinical application the robotic system was used for the exact placement of implants to retain facial prostheses. In addition, the facial prostheses were fabricated using a rapid prototyping method and by mirroring the other side of the patient's face. In the paper the authors' experiences integrating the system into clinical routine are described. It became evident that only a careful optimization of each step of the intervention, starting with image acquisition and the patient fixation through the planning and the intraoperative execution by the robot leads to a significant advantage.
\end{abstract}

\section{Introduction}

Surgery is a relatively new and a rapidly growing field of application for robotics technology. Robots can be used to enhance the accuracy and the dexterity of a surgeon, decrease the factor of human tremble and amplify or reduce the movements and/or forces applied by the surgeon. Especially in fields of surgery where the human hand is the limiting factor for further optimization of surgical techniques - neurosurgery, orthopedic and maxillofacial surgery - robotics technology can be applied.

The drilling or shaping of bone structures is of great importance in surgery. In contrast to the situation with soft tissue, a static model of bone structures derived from CT images can be used. Currently, these operations are carried out by surgeons free hand. In the vicinity of sensitive regions, manual handling can lead to complications due to inaccuracies or shattering of the drill or shaper. Especially in maxillofacial surgery the accuracy of an intervention is of paramount importance due to the high social and aesthetic impact of the face. Therefore, a high accuracy is desirable when the surgeon 
positions and moves drills or shapers. Additional difficulties in maxillofacial surgery are the restricted access to the bone structures through small incisions, the swelling of tissue during the intervention, and the close proximity of vital organs or structures. In this paper the first clinical application and experiences in integrating this system into the intervention process are described. It will be shown that the application of a robotic system is only successful if a number of infrastructure problems such as fixation, image acquisition and planning have been solved.

\section{State of the Art}

The commercially available surgical robots can be distinguished by their different control strategies:

- Automatic systems: are used for the autonomous performance of a preplanned intervention. Examples are the shaping of cavities for total hip replacement [1] and the shaping of surfaces at the knee joint [2]. Because of the high safety requirements for automatic systems, the application only makes sense for complex interventions where the robot can carry out a part of the intervention without any interaction with the surgeon. Commercial systems available are Robodoc by ISS and Caspar by Orto Maquet.

- Telemanipulation systems: are used for minimally invasive interventions where the surgeon has no direct access to the operation site. The systems reduce the human tremble factor and allow movements or forces to be scaled up or down. Currently systems are available for positioning cameras (Aesop by Computer Motion [3]) and instrument-carrying telemanipulators (Zeus by Computer Motion and da Vinci by Intuitive Surgical [4]).

- Interactive controllable systems: are a mixture of the two system approaches described above. The surgeon is not spatially separated from the patient. Additionally, during the entire intervention the surgeon can intercept the course of the operation. Apart from the systems developed by the authors [5][6] up to now only systems for positioning operation microscopes have been developed (SurgiScope by Jojumarie and MKM by Zeiss).

A direct human-machine coupling is the basis for the interactive control of a robot. Methods are the attachment of input devices directly at the manipulator (e.g. the space mouse at the MKM and joysticks at the SurgiScope) or the insertion of a force/torque sensor between the hand axis and the instrument to guide a robot. In [7] this approach is used for teaching positions and trajectories with industrial robots. A similar approach is used in [8] for the teaching of registration markers at the patient. This architecture does not allow the distinction between forces applied by the operator and forces caused by interactions with the environment or the patient. Therefore in [9] and [10] the manipulators have been supplied with two force/torque sensors - one between tool and manipulator and one between manipulator and operator. Disadvantages of this extension are increased costs and - especially inconvenient in surgery - the necessary modification of the mounted surgical instrument or a different use of the instrument. 
The authors use an architecture with only one force/torque sensor. The main advantage for the surgeon is the use of unmodified surgical instruments in the usual manner. A similar approach has been used in [11] for an experimental system.

Apart from the authors' system, only two other systems are being developed currently in maxillofacial surgery. The system described in [12] consists of a passive manipulator used for positioning instruments. The disadvantage of passive systems is the time consuming positioning procedure required with them and the impossibility of moving along desired trajectories with force constraints. The other system [13] is an experimental automatic system that is not designed for interaction with the surgeon. Such systems seem unsuitable for actual application in maxillofacial surgery.

\section{Description of the Treatment}

An ear prosthesis is an alternative to plastic reconstructive surgery if the whole ear is involved. Ear anomalies can be congenital in origin or the result of tumor surgery or trauma. The best method for retaining the prosthesis is the insertion of implants to carry the ear prosthesis. Usually a two-stage surgical intervention is carried out. In the first stage the implants are inserted in the area of the outer helix of the artificial ear. The implants are covered by skin and after a three week healing phase are uncovered. A supraconstruction is then fixed through the skin. Using impressions of the supraconstruction the artificial ear is modeled out of silicon in a manual procedure. Usually it takes at least three months before the patient gets his facial prosthesis.

To shorten this procedure and to enhance the quality of the intervention, the authors have developed a robot-assisted procedure. Fig. 1 shows the workflow of the whole intervention. The following steps will be described in detail in this paper:

- Image Acquisition and Fixation: In contrast to navigation systems, robotic systems require stable fixation of the patient. An additional stabilization of the operating table, an individual head rest and an intermaxillary splint were used for this purpose. The patient's position was also fixated for the CT scan. In this way the quality of the image data was improved.

- Planning and Fabrication of the Facial Prostheses: The insertion of craniofacial implants for the secure retention for facial prostheses must be carried out according to functional and esthetic considerations. The amount of available bone and the soft tissue in the prospective implant position must be taken into consideration to preclude any damage to important anatomic structures. The prosthesis is manufactured from the planning data using rapid prototyping before the intervention.

- Robot-Assisted Placement of the Implants: At the end of the planning and fabrication process the robotic system is used for exact drilling at the preplanned positions and the insertion of the implants in a way that the implants are parallel and have the defined distance. Up to now, navigation techniques and operation templates have been used for this. The implant site can be located with navigation, but an exact freehand positioning of the implants is not possible. Templates are difficult to keep fixed and do not afford accurate guidance while drilling. 


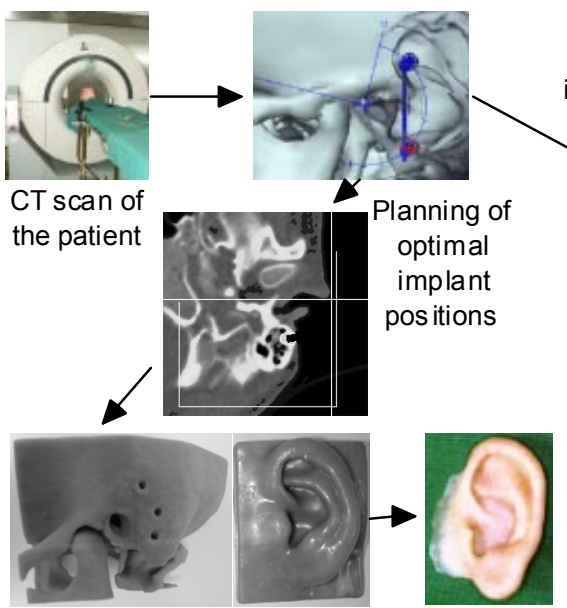

Rapid prototyping models of the bone surface and the mirrored ear, prepared facial pros thesis
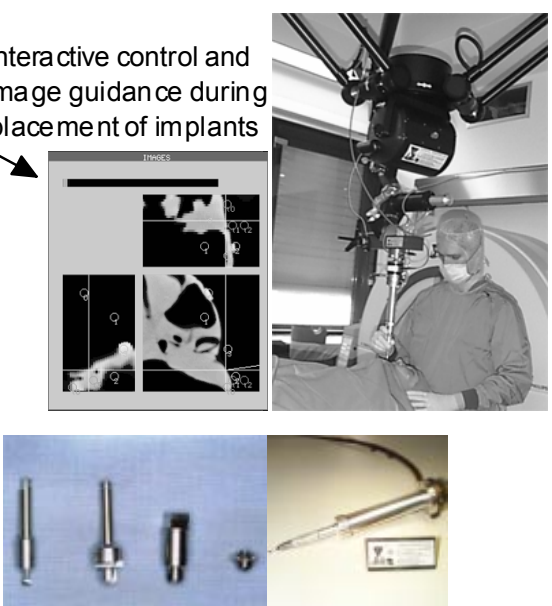

Surgical Instruments and fixations

Fig. 1. Workflow for the fabrication of a facial prosthesis using rapid prototyping and implant insertion using a robot system.

\subsection{Image Acquisition and Fixation}

To achieve optimal image quality for the preoperative planning and near-to-identical conditions during data acquisition and the actual intervention, the patient's position was fixated during both steps with the same fixation system and at the same OR table. The fixation system consisted of an individual polyurethane foam dish and a custom intermaxillary splint (Fig. 2. The foam provided a large contact surface for the patient's soft tissue and allowed a rough positioning of the patient. The intermaxillary splint provided rigid contact between the patient's head and a lockable hydraulic arm. This arm held the patient rigidly to the OR table. A reference frame of the navigation system was also attached to the splint.

a)

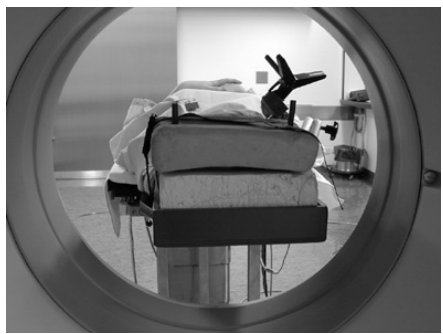

b)

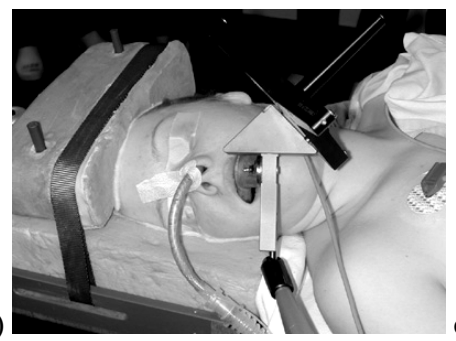

c)

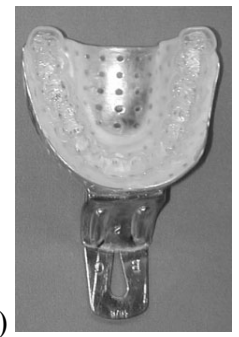

Fig. 2. Fixation system for the robot assisted intervention. The system consists of a individual head rest and a arm rigidly holding the patient by means of an intermaxillary splint. a) Fixation during CT scan, b) during the intervention, and c) custom-made intermaxillary splint. 
The registration was done via markers around the operation site. These markers were fixed within a mold of silicon and not on the patient's skin. In this way errors due to movement of markers on the skin were minimized. After the CT scan the positions of the markers relative to the reference frame of the navigation system were determined. Using the marker positions within the image coordinate system the registration matrix was calculated with a point matching algorithm. Because the repositioning accuracy achieved with the intermaxillary splint was sufficiently high, no further registration was necessary during the intervention, but rather the robot controller received the registration matrix from the planning tool.

\subsection{Planning and Fabrication of the Facial Prostheses}

In the planning tool Fig. 3 ) the implant positions and orientation were determined preoperatively according to available bone, soft tissue situation and esthetic considerations. Most important for high initial implant stability and to avoid infections is that the implants must not penetrate into mastoid cells Fig. 3 ). Bone thickness at the site must be adequate. Another problem is determining the aesthetically optimal position and orientation of the ear and therefore of the implants. Because only the necessary images are taken during the CT scan, the spatial orientation is very difficult. To solve this problem the anaplastologist preparing the facial prosthesis defined the optimal ear and implant positions before the CT scan. At these positions metal threads were fixed beneath the mold. During the planning the tool visualized these positions (Fig. 3 ) and the surgeon could position the implants accordingly.

a)

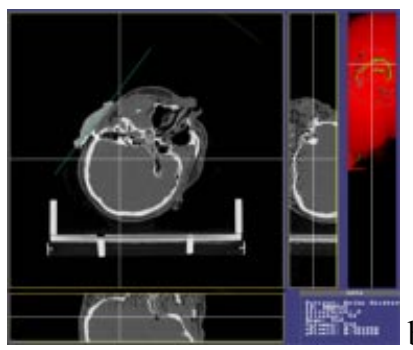

b)

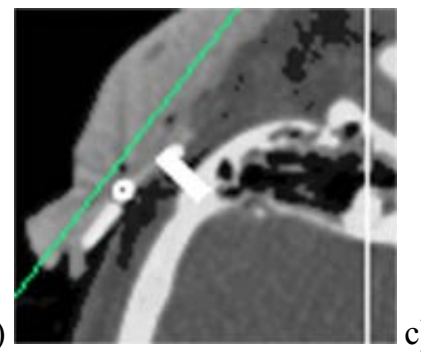

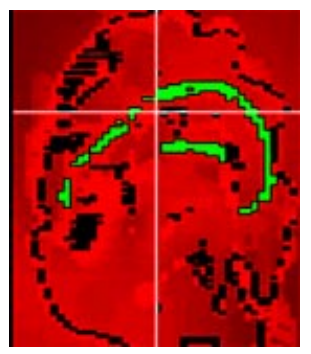

Fig. 3. a) Planning tool with three slices and a rendered $3 D$ view right hand side. b) Operation area in the axial view with an inserted implant. On the right of the implant air-filled mastoid cells can be seen. c) Part of the 3D view with the position aids (aesthetically preferred implantation positions and outer helix of the ear).

After the implant and marker positions had been defined, the data together with a region of interest were exported to the robot controller for execution and the modified images were exported to a surface model in STL format (Standard Transformation Language). Rapid prototyping was used to fabricate a model of the operation area with the implants (Fig. 4i) and the mirror-image replica of the patient's healthy ear (Fig. b). These two pieces were to enable the anaplastologist to produce the silicone ear prosthesis prior to surgery. However, due to errors in the slice distance of the CT (slice distance of $2 \mathrm{~mm}$ can differ by up to $20 \%$; [14]), errors during the segmentation 
of the skin, and metal artifacts from the fixation system and the patient's dental fillings, the quality of this first surface model ( $a$ and $b$ ) was too poor for use, and an alternative approach was necessary. An exact replica of the surface skin of the defect site was then made from a mold. This plaster model was registered, and two parallel implants and magnets were inserted into it in a robot assisted model operation (Fig. 4c). With this model and with the mirror-image replica of the healthy ear, the anaplastologist was able to manufacture the facial prosthesis before the intervention.

a)

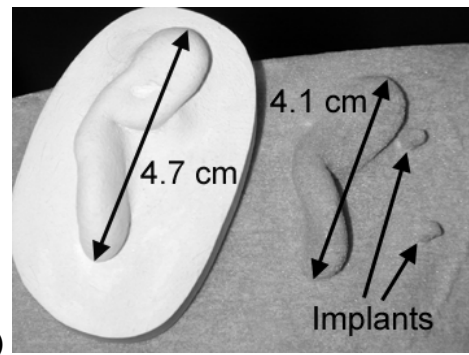

b)

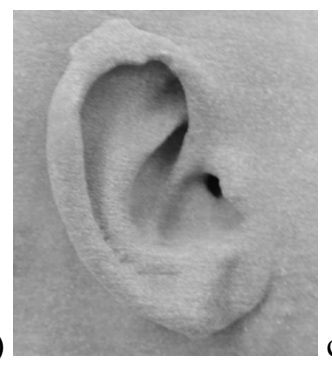

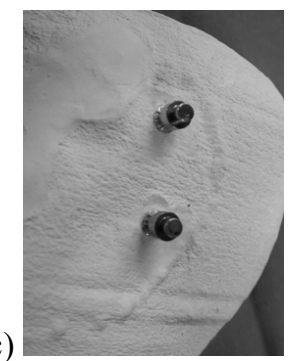

Fig. 4. a) Differences between the plaster cast of the replaced ear part and the stereolithographic model. The size of the ear parts deviate by $6 \mathrm{~mm}$. b) Reconstruction errors caused by noise and artifacts in the image data. At the top of the ear an erroneous structure appeared. c) Successful plaster model with inserted implants.

\subsection{Robot-Assisted Placement of the Implants}

The SurgiScope (originally by Elekta IGS) was used as the basis for the developing a robotics system for maxillofacial surgery. The SurgiScope system was designed to position microscopes for neurosurgery. For invasive applications, the controller software and the tools were changed [5][6]. The robotics system consists of a parallel manipulator (Delta-3 kinematic), an infrared navigation system (IGT FP 3000), a control cabinet with a computer for the navigation system and a control computer for the manipulator. A tool system with a mechanism for changing tools and a forcetorque sensor were constructed. For maxillofacial surgery, a standard drilling machine was mounted on the robot.

The robotic system differs from other systems through its completely interactive control. During the entire intervention the robot behaves passively, and moves only if the surgeon grasps and applies force to the drill. Through specially designed control schemes the surgeon can operate the instrument as usual except in cases where he/she wants to work with more precision (e.g. find a predefined position or drill along a defined axis). In these cases the robotic system controls the necessary position and orientation degrees and the surgeon can only interactively control the remaining degrees (e.g. rotations around the $z$-axis of the drill). Currently the system can be interactively moved to target positions along trajectories and within predefined volumes. The information from the planning system is transferred by a file which also contains the image information of a region of interest. This image information is used on the 
one hand for intraoperative visualization and on the other hand for autonomous derivations of control commands and to update the patient model.

\section{Results and Conclusions}

Initial phantom experiments with the system showed a positioning accuracy of $0.5 \mathrm{~mm}$ $\pm 0.4 \mathrm{~mm}(N=40)$. The relative accuracy was determined during the placement of implants in plaster bodies. The mean deviation from the defined distance was $0.2 \mathrm{~mm}$ $\pm 0.5 \mathrm{~mm}$ and the deviation from the parallel orientation was $0.6^{\circ} \pm 0.5^{\circ}(N=9)$. The system's handling and accuracy under real conditions were assessed in the clinical evaluation.

Robot assisted implantation was carried out on 13 patients. In total 30 craniofacial implants (Entific Medical Systems) were inserted in out-patient surgery under general anesthesia. All patients had congenital ear defects which were rehabilitated with implant-anchored silicone ear prostheses. Three implants were inserted in the first four patients, with one serving in each case as a reserve. In the following nine patients, given the positive experience of the first operations, only two implants were inserted. No intraoperative injuries with opening of the mastoid cells or damage to the venous sinuses at the base of the cranium or dura mater encephali occurred. With the exception of one patient whose intermaxillary splint disconnected due to insufficiently deep anesthesia, all targeted positions were reached with an accuracy of about $\pm 1 \mathrm{~mm}$. For this single patient new implant positions were determined intraoperatively, but the preoperatively created prosthesis did not fit. In the other cases the preoperatively manufactured ear prostheses were fitted (Fig. 5), but these patients were advised not to wear the prosthesis constantly for the first three months to avoid disturbing the osseointegration.

a)

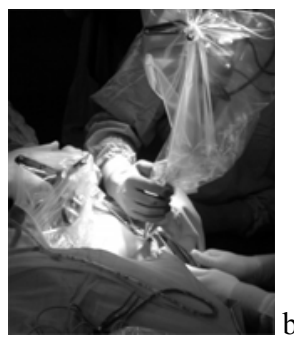

b)

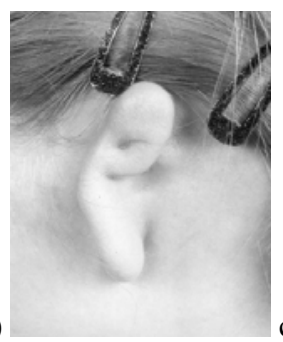

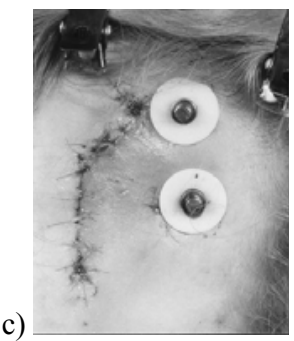

d)

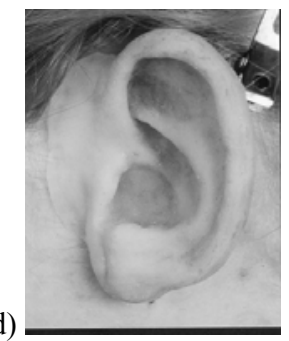

Fig. 5. Clinical application of the interactive controlled robot system (a) and result in a patient: b) preoperative, c) postoperative, d) facial prosthesis fixed by the implants.

The robot assisted implantation presented here and the new concept of preoperative manufacture of the prosthesis are as a whole more complex than manual implant insertion. They demand much more precise preoperative planning, intraoperative execution, and a careful integration into the usual course of the intervention. Nonetheless, a trained team which takes all potential sources of error into consideration can deliver 
excellent esthetic and functional results. Furthermore, the patient's rehabilitation time is shortened, because with the exception of one patient, all patients could wear the new prosthesis directly after the intervention. The new manufacturing process for the facial prosthesis saves the anaplastologist one day's modeling time, and the patient is spared hours in repeated fittings. The positive clinical experience and the accuracy attained in the insertion of craniofacial implants to retain ear prostheses is good cause to apply this robot system for insertion of implants in other areas of the head or as the next planned application to support the surgeon shaping and inserting pedicle screws in spinal surgery.

\section{References}

1. Taylor, R. H. et al:: An Image-Directed Robotic System for Precise Orthopaedic Surgery. IEEE Trans. o. Robotics and Automation, Vol.10, No.3 (1994), pp. 261-75.

2. Kaiser, C.: Einführung in die technischen Grundlagen des totalen Kniegelenkersatzes mit CASPAR. In Maßberg, W., G. Reinhart, M. Wehmöller (Ed.), Neue Technologien in der Medizin, Shaker Verlag (2000).

3. Jacobs LK, Shayani V, Sackier JM: Determination of the learning curve of the AESOP robot. Surg Endosc, Jan; 11(1) (1997), pp. 54-5.

4 Guthart, G.S., J.K. Salisbury: The Intuitive telesurgery system: overview and application. ICRA IEEE Int. Conf. on Robotics and Automation (2000).

5 Lueth, T.C. et al.: A Surgical Robot System for Maxillofacial Surgery. IEEE Int. Conf. on Industrial Electronics, Control, and Instrumentation (IECON), Aachen, Germany, Aug. 31Sep. 4, (1998) 2470-2475.

6 Hein, A.: Eine interaktive Robotersteuerung für chirurgische Applikationen. Ph.D. Thesis. Fortschritt-Berichte, Nr. 195, VDI-Verlag, Düsseldorf (2000).

7 Hirzinger, G., K. Landzettel: Sensory feedback structures for robots with supervised learning. ICRA IEEE Int. Conf. on Robotics and Automation (1985), pp. 627-35.

8 Kazanzides, P. et al.: Force sensing and control for a surgical robot. ICRA IEEE Int. Conf. on Robotics and Automation (1992), pp. 612-7.

9 Kosuge, K., Y. Fujisawa, T. Fukuda: Mechanical System Control with Man-MachineEnvironment Interactions. ICRA IEEE Int. Conf. on Robotics and Automation (1993), pp. 239-44.

10. Taylor, R. et al:: A steady-hand robotic system for microsurgical augmentation. MICCAI Int'l Conf. on Medical Image Computing and Computer-Assisted Intervention (1999), pp. 1031-41.

11. Ho, S. C. et al.: Force control for robotic surgery. ICAR IEEE Int'l. Conf. on Advanced Robotics (1995), pp. 21-31.

12. Cutting, C., F. Bookstein, R. Taylor: Applications of simulation, morphometrics and robotics in craniofacial surgery. In Taylor, R. H., S. Lavallee, G. C. Burdea, R. Mösges (eds.), Computer-integrated surgery: technology and clinical applications, MIT Press, (1996) 641662.

13. Bohner, P. et al:: Operation planning in cranio-maxillo-facial surgery. Medicine Meets Virtual Reality 4 (MMVR4'96), San Diego, California, (1996).

14. Albrecht, J., T.C. Lueth, A. Hein, J. Bier: Measurement of the Slice Distance Accuracy of the Mobile CT Tomoscan. CARS 2000, Computer Assisted Radiology and Surgery, (2000). pp. 651-5. 